\title{
Estudio de la Gestión de la Seguridad y Salud en el sector construcción valenciano Study of Safety and Health Management in the construction sector of Valencia
}

\author{
José Luis Fuentes-Bargues ${ }^{\mathrm{a}}, \mathrm{M}^{\mathrm{a}}$ Carmen González-Cruz ${ }^{\mathrm{b}}$, Cristina González-Gaya ${ }^{\mathrm{c}}$, \\ Naiara García-Arambarri ${ }^{\mathrm{d}}, \mathrm{M}^{\mathrm{a}}$ del Prado Díaz de Mera-Sánchez ${ }^{\mathrm{e}}$

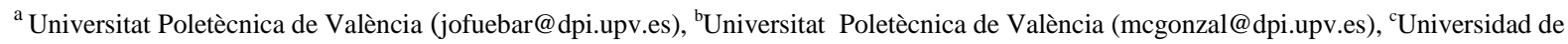 \\ Educación a Distancia (cgonzalez@ind.uned.es), ${ }^{\mathrm{d} U n i v e r s i t a t ~ P o l e t e ̀ c n i c a ~ d e ~ V a l e ̀ n c i a, ~}{ }^{\mathrm{e} U n i v e r s i d a d ~ R e y ~ J u a n ~ C a r l o s ~ I ~}$
}

\begin{abstract}
Resumen-La construcción es un sector que presenta un alto nivel de siniestrabilidad debido a sus características propias (alto componente de mano de obra y que se desarrolla al aire libre) y por sus características organizativas, como los ritmos de trabajo, las metodologías de ejecución y la elevada subcontratación. La gestión de la Seguridad y Salud es un factor determinante a la hora de obtener mejoras en la ejecución de los trabajos y menores tasas de accidentes. En este trabajo se realiza un estudio del nivel actual de gestión de la seguridad y salud en el sector construcción en Valencia (España) a partir de una encuesta realizada a técnicos con experiencia en el sector. Los principales resultados obtenidos son que el tamaño de la empresa es un factor clave para la seguridad y salud, tanto en los aspectos de formación como en la gestión de la documentación de Seguridad y Salud. Los resultados muestran también cómo a pesar del tiempo transcurrido desde las últimas aportaciones normativas, tales como el Libro de Subcontratación o el Registro de Empresas Acreditadas, las empresas tienen un alto desconocimiento de la misma.
\end{abstract}

Palabras clave - Seguridad y Salud, Gestión, Construcción, Valencia

\begin{abstract}
Construction is a sector with a high accident rate due to its own characteristics (high component of labor and develops outdoor) and its organizational characteristics as working patterns, methods of implementation and higher outsourcing. Management of Health and Safety is a determining factor in obtaining best execution of work and lower rates of accidents. This paper presents a study of the current level of Health and Safety Management in the construction sector in Valencia (Spain) from a survey of a group of technicians with experience in the sector. The main results are the size of the company is an important key to health and safety, both in terms of training as in the management of documentation. It also reflects how, despite the time elapsed since the latest regulations, such as the Book of Outsourcing or Accredited Companies Registry, companies have a high rate of ignorance.
\end{abstract}

Index Terms - Health and Safety, Management, Construction, Valencia

\section{INTRODUCCIÓN.}

Las obras de construcción son lugares de trabajo complejos

(a) Departamento de Proyectos de Ingeniería, Universitat Politècnica de València. Departamento de Ingeniería Química (jofuebar@dpi.upv.es).

(b) Departamento de Proyectos de Ingeniería, Universitat Politècnica de València.

(c) Departamento de Ingeniería de Construcción y Fabricación, ETSII, UNED. (d) Máster en Dirección y Gestión de Proyectos, Universitat Politècnica de València.

(e) Departamento de Tecnología Química y Energética, Tecnología Química y Ambiental y Tecnología Mecánica y Química Analítica, Universidad Rey Juan Carlos I. y en muchas ocasiones inseguros por diversos motivos: su condición de trabajo al aire libre, la dependencia de los factores metereológicos, los ritmos de trabajo, las metodologías de ejecución, el alto componente de mano de obra y su elevada subcontratación (Pellicer-Armiñana, 2003; Fuentes-Bargues et al., 2015).

La alta tasa de accidentes en las obras de construcción las convierte en uno de los lugares de trabajo más peligrosos, 
tanto a nivel español (Sesé et al., 2002; Camino-López et al., 2008, Pérez-Alonso et al., 2012; López-Alonso et al., 2015) como a nivel europeo (Ros-Serrano et al., 2013; Ulubeyli et al., 2015) y a nivel mundial (Huang et al., 2003; Teo et al., 2005; Loosemore et al., 2007; Esteban-Gabriel et al., 2015).

Muchos de los accidentes en el sector construcción son causados por errores de organización, planificación y control (HSE, 1988; López-González et al., 2012), si bien una mayor implicación de los altos niveles de dirección de las empresas y una mejora sobre los sistemas de gestión son líneas de actuación para lograr una reducción del número de accidentes (Mattila et al., 1994; Teo et al., 2005; Loosemore et al. 2007).

Otra de las características del sector construcción es la existencia de una elevada subcontratación, y no solo a un nivel, sino a varios niveles, en especial los trabajos que únicamente conllevan mano de obra (Sesé et al., 2002; Teo et al., 2005; $\mathrm{Ng}$ et al., 2010). Esto exige una continua colaboración entre contratistas y subcontratistas, ya que la falta de comunicación, control y coordinación (Debrah et al., 2001) junto con el inadecuado comportamiento de las empresas subcontratadas son una de las principales causas del fallo del proyecto y de la siniestrabilidad (Ardite et al., 2005; Camino-López et al., 2008).

Algunos estudios señalan que el tamaño de la empresa es un factor de gran influencia en la gestión de la seguridad y salud, como los realizados en Australia by Lin and Mills (2001), en China by Tam et al. (2004) y en Gran Bretaña por Reynolds et al. (2008). Las pequeñas empresas tienen un alto nivel de siniestrabilidad cuando se comparan con las grandes empresas.

En España se han realizado trabajos sobre Seguridad y Salud en construcción desde diversos enfoques. Cabe destacar los trabajos de Sesé et al. (2002) que describe el marco regulatorio y la situación en el año 2002. Posteriormente, Camino-López et al. (2008) analizaron los accidentes que tuvieron lugar en las obras de construcción y su severidad de acuerdo a dieciocho variables. Ellos concluyeron entre otros aspectos, en que los trabajadores con contratos temporales tienen una mayor probabilidad de sufrir accidentes graves o mortales y que la probabilidad de que un accidente sea severo, es mayor en los accidentes que se producen en las empresas con menos de veinticinco trabajadores. Pérez-Alonso et al. (2012) en su investigación sobre el subsector construcción de invernaderos en el sureste español afirmaron que las evaluaciones realizadas por las empresas eran deficientes porque en muchas ocasiones las empresas no sabían cómo llevar a cabo tales evaluaciones.

Ros-Serrano et al. (2013) señala la importancia de la figura del Coordinador de Seguridad y Salud, así como de sus conocimientos y habilidades, en los proyectos de construcción. López-González et al. (2013, 2014), en su estudio sobre el sector construcción riojano, afirman que los Estudios de Seguridad y Salud, la definición de las etapas de la ejecución, los aspectos económicos y una mejor definición en el diseño, debían ser mejorados. En el año 2014, Fuentes et al. (2014) reflejaron sobre una pequeña muestra de proyectos de construcción en Valencia, la falta de conocimiento y formación en Seguridad y Salud en las empresas con menos de cinco trabajadores.

El objetivo de este artículo es el estudio del nivel actual de gestión de la seguridad y salud en el sector construcción en Valencia (España) a partir de una encuesta realizada a técnicos que han desarrollado su actividad laboral en empresas de construcción en los últimos años. El artículo se estructura en cinco secciones. En primer lugar la Introducción, en segundo lugar una sección donde se describe el marco normativo y se definen los principales ítems sobre Seguridad y Salud desarrollados posteriormente. En la tercera sección se presenta la metodología seguida durante la investigación. En la cuarta sección se presentan los resultados y en la quinta sección se reflejan las Conclusiones.

\section{El MARCO NORMATIVO SOBRE SEGURIDAD Y SALUD EN EL SECTOR CONSTRUCCIÓN.}

En el año 1995 se traspuso la Directiva 89/391/CEE (Unión Europea 1989) a la normativa española, la Ley 31/1995 sobre Prevención de Riesgos Laborales (España 1995), convirtiéndose en la norma de referencia en materia preventiva. A partir de ésta se elaboraron normativas específicas en cada sector, con el objetivo de establecer un adecuado nivel de protección de la seguridad y salud de los trabajadores frente a los riesgos derivados de las condiciones de trabajo.

En el año 1997, se aprobó el Real Decreto 39/1997 que desarrolló el Reglamento de los Servicios de Prevención (España 1997a). Se define cómo servicio de prevención al conjunto de medios humanos y materiales necesarios para realizar las actividades preventivas. Se distinguen cuatro tipos: el propio empresario, los servicios de prevención propios, los servicios de prevención ajenos y los servicios de prevención mancomunados.

Ese mismo año se desarrolló el RD 1627/1997 (España 1997b) por el cual se establecieron las disposiciones mínimas sobre seguridad y salud en las obras de construcción. En esta norma se desarrollaron las obligaciones y responsabilidades de cada una de las figuras que participan en el proceso constructivo, entre ellas la figura del Coordinador de Seguridad y Salud (CSS). Entre las funciones de los CSSs están la supervisión y coordinación entre contratistas, subcontratistas y trabajadores autónomos, así como la aprobación del Plan de Seguridad y Salud (PSS). Se establece la obligatoriedad de la existencia de un Libro de Incidencias (LI) en cada centro de trabajo cuyo fin será el control y seguimiento del PSS durante la ejecución de los trabajos. 
Las diferentes modificaciones de la normativa de seguridad y salud han introducido nuevos requisitos con objeto de reducir los altos índices de siniestrabilidad. Entre estas modificaciones se ha de destacar el RD 171/2004 (España 2004) que creó la figura del Recurso Preventivo (RP), por la cual el contratista (y los correspondientes subcontratistas) tienen que nombrar a personal propio con capacidad suficiente y medios necesarios para poder vigilar el cumplimiento de las medidas preventivas y comprobar la eficacia de éstas. En esta misma norma se potenció la necesidad de coordinación de actividades desde el punto de vista de seguridad y salud entre las empresas contratistas y subcontratistas presentes en una obra.

Posteriormente se elaboraron la Ley 32/2006, reguladora de la subcontratación en el Sector de la Construcción (España 2006), y el Real Decreto 1109/2007, que desarrolla la citada ley (España 2007), donde se delimitan los grados y las condiciones para la subcontratación. Se limita el número de cadenas de subcontratación y se regula la documentación a presentar por parte de las empresas contratistas y subcontratistas, creando las siguientes figuras documentales:

- El Libro de Subcontratación (LS), de obligada tramitación para el contratista principal, que deberá estar presente en obra y donde se registrarán todos los datos referentes a las subcontrataciones realizadas.

- El Registro de Empresas Acreditadas (REA), donde todas las empresas que pretendan ser contratadas o subcontratadas para trabajos en una obra de construcción deberán estar inscritas.

- La Tarjeta Profesional de la Construcción (TPC), documento mediante el cual se acreditan, entre otros datos, la formación recibida por el trabajador del sector en materia de prevención de riesgos laborales, su categoría profesional, y sus periodos de ocupación de las distintas empresas en las que haya ejercido su actividad.

\section{MÉTODO.}

El método utilizado en este estudio se basa en una encuesta sobre varios aspectos de gestión de Seguridad y Salud, que fue enviada a una muestra de técnicos con experiencia en el sector construcción valenciano. La encuesta se dividió en tres bloques (Tabla 1). El primer bloque corresponde a datos generales de los participantes (sexo, edad y nivel de estudios) y de las características de la empresa, tales como el subsector donde se desarrolla la actividad, el tamaño (número de trabajadores) y la formación en Seguridad y Salud del empresario o responsable de la empresa.

El segundo bloque consiste en una serie de cuestiones sobre la organización de la Seguridad y Salud en la empresa, desarrollando aspectos como la integración de la prevención de riesgos laborales en la política de la compañía, el sistema de organización de la actividad preventiva, la existencia de Delegados de Prevención, la existencia de un Comité de Seguridad y Salud y el Registro de Empresas Acreditadas.

El tercer bloque corresponde a la gestión de la documentación de Seguridad y Salud durante las obras. Las cuestiones indagan sobre cómo se prepara la documentación de Seguridad y Salud (PSS, Libro de Subcontratación y otros trámites), así como sobre el nombramiento de los recursos preventivos, tanto de las empresas contratistas como de las subcontratistas.

\section{RESUlTADOS.}

La encuesta se envío a una muestra de cien técnicos con experiencia en el sector construcción que habían desempeñado las funciones de Coordinador de Seguridad y Salud, Director de Obra o Jefe de Obra durante su carrera profesional. La muestra fue elaborada por los autores del presente artículo e incluía profesionales de todas las titulaciones relacionadas con el sector, tales como Arquitecto, Arquitecto Técnico, Ingeniero Industrial, Ingeniero Técnico Industrial, Ingeniero de Caminos, Canales y Puertos, Ingeniero de Obras Públicas, etc.

La encuesta fue enviada por correo electrónico en Mayo de 2015. Se realizaron varias iteraciones con el objetivo de lograr el máximo número de respuestas y la recogida de datos finalizó a final de Julio de 2015. Se recogieron veintiocho respuestas, veintisiete válidas y una incompleta. Se presentan en este apartado los principales resultados del estudio.

La muestra recibida esta compuesta por siete mujeres y veinte hombres, con edades entre los 25 y los 72 años. El nivel de estudios de los encuestados es de siete Doctores, once técnicos nivel Master, un Arquitecto y ocho Arquitectos Técnicos e Ingenieros Técnicos.

Se indicó a los encuestados que respondieran a las diferentes cuestiones en referencia a la empresa actual o sobre la última empresa donde hubieran desempeñado su carrera profesional. Las empresas se clasificaron según su tamaño, en este caso por el número de trabajadores, distinguiéndose cuatro escalones: Micro, Pequeña, Mediana y Grande. El grupo de Microempresas comprende las empresas entre 2 y 10 trabajadores (11 empresas), el grupo Pequeña empresa comprende las empresas entre 11 y 25 trabajadores (5 empresas), el tercer grupo, Mediana empresa, comprende a aquellas entre 26 y 50 (4 empresas) y finalmente las empresas con más de 50 trabajadores (7 empresas) se consideraron Grandes Empresas.

Respecto al subsector donde la empresa desarrolla su actividad, catorce empresas la desarrollan en el subsector Edificación, dos empresas en el subsector Obra Civil y once empresas en ambos subsectores. 
Otro de los datos iniciales recogidos fue la formación en materia de Seguridad y Salud del empresario o responsable de la empresa, cuatro disponen de Nivel Básico, seis disponen de
Nivel Intermedio y diecisiete disponen de Nivel Superior.

El segundo bloque de la encuesta tiene el objetivo de analizar la organización de las empresas en materia de

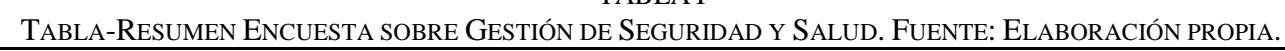

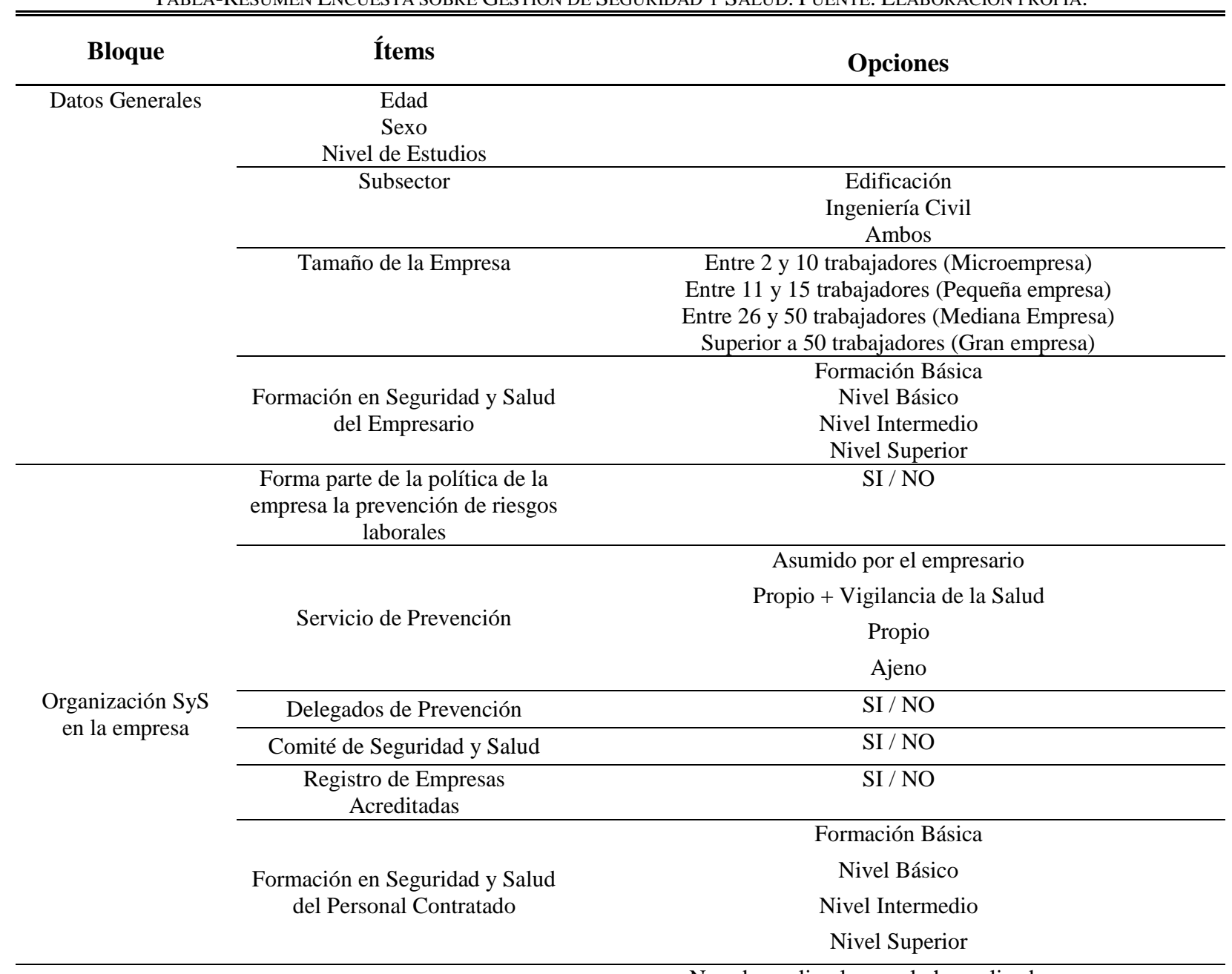

No sabe realizarlo o no lo ha realizado nunca

Plan de Seguridad y Salud

Elaborado por sus propios medios

Subcontratado (técnicos externos, servicio de prevención, asesoría, etc.)

No sabe diligenciarlo o no lo ha diligenciado nunca

Elaborado por sus propios medios

Libro de Subcontratación

Subcontratado (técnicos externos, servicio de prevención, asesoría, etc.)

Gestión de la documentación de SyS en obra
No sabe realizarlos o no los ha realizado nunca

Elaborado por sus propios medios

Trámites con la Administración

Subcontratado (técnicos externos, servicio de prevención, asesoría, etc.)

Está presente o no en obra.

Se nombra por iniciativa propia o por iniciativa del Coordinador de Seguridad y Salud.

Posee o no la formación adecuada.

Está presente o no en obra.

Recurso Preventivo Subcontratista
Se nombra por iniciativa propia o por iniciativa del Coordinador de Seguridad y Salud.

Posee o no la formación adecuada. 
TABLA II

TiPO DE SERVICIO DE PREVENCIÓN EN FUnCIÓN DEL TAMAÑo DE EMPRESA. FuENTE: ElABORACIÓN PROPIA

\begin{tabular}{|c|c|c|c|c|}
\hline \multirow{2}{*}{$\begin{array}{l}\text { Tipo de Servicio de } \\
\text { Prevención }\end{array}$} & \multicolumn{4}{|c|}{ Tamaño de la empresa } \\
\hline & Micro & Pequeña & Mediana & Grande \\
\hline $\begin{array}{c}\text { Asumido por el } \\
\text { empresario }\end{array}$ & 6 & 0 & 1 & 1 \\
\hline Propio & 2 & 0 & 0 & 1 \\
\hline $\begin{array}{c}\text { Propio + Vigilancia } \\
\text { de la salud }\end{array}$ & 1 & 2 & 1 & 2 \\
\hline Ajeno & 2 & 3 & 2 & 3 \\
\hline
\end{tabular}

Seguridad y Salud. El primero de los ítems a estudiar fue si en la política empresarial se incluían objetivos o aspectos relativos a prevención de riesgos laborales. A excepción de dos empresas (una perteneciente al grupo Microempresas y otra al grupo Pequeña Empresa), todas las empresas los incluían.

El segundo ítem que se analizó fue la organización de la prevención en la empresa, en este caso el servicio de prevención. Se plantearon cuatro posibles respuestas: servicio de prevención propio (3 empresas), servicio de prevención ajeno (10 empresas), prevención asumida por el propio empresario ( 8 empresas) y sistema de prevención propio con la externalización de la vigilancia de la salud de los trabajadores (6 empresas).

Si se analiza la organización de la prevención respecto al tamaño de la empresa (Tabla 2), se muestra como la forma de la organización de la prevención más utilizada por parte de las pequeñas, medianas y grandes empresas es la contratación de servicios de prevención ajenos. En el grupo de Microempresas destaca la asunción de la actividad preventiva por parte del empresario.

Los siguientes aspectos que se analizaron en este bloque fueron relativos a la existencia de Delegados de Prevención y de Comité de Seguridad y Salud en la empresa. Dado que se trata de requisitos normativos obligatorios a partir de 50 trabajadores se analiza el grupo correspondiente a Grandes empresas y los resultados muestran que en dos de las empresas no existen ni Delegados ni Comité, y en otras dos de las empresas existen Delegados pero no Comité. A priori, la no existencia de Delegados ni de Comité de Seguridad y Salud en estas empresas parece extraño, ya que se trata de un requisito normativo desde el año 1995, y la explicación podría ser el desconocimiento por parte de los trabajadores de quienes son los Delegados de Prevención y quién conforma el Comité de Seguridad y Salud de la empresa. En cualquier caso la no existencia o el desconocimiento por parte de los trabajadores son aspectos que indican una organización deficiente en materia de prevención de riesgos laborales.

Finalmente dentro de este bloque se preguntó si las empresas disponían de Registro de Empresas Acreditadas (REA), y se obtuvo que 12 de las 25 empresas no disponían de él. De estas doce empresas, siete de ellas pertenecen al grupo Microempresas.

En cuanto al nivel máximo de formación de los trabajadores en prevención de riesgos laborales, en ocho empresas el nivel es básico, en cinco empresas el nivel es intermedio y en catorce el nivel es superior. Si se compara el nivel de formación en función del tamaño de empresa (Figura 1) no hay una pauta establecida, aunque si es destacable la formación de nivel superior en microempresas. Si se compara la formación del personal en función de la formación del empresario (Figura 2) si que se muestra una relación clara

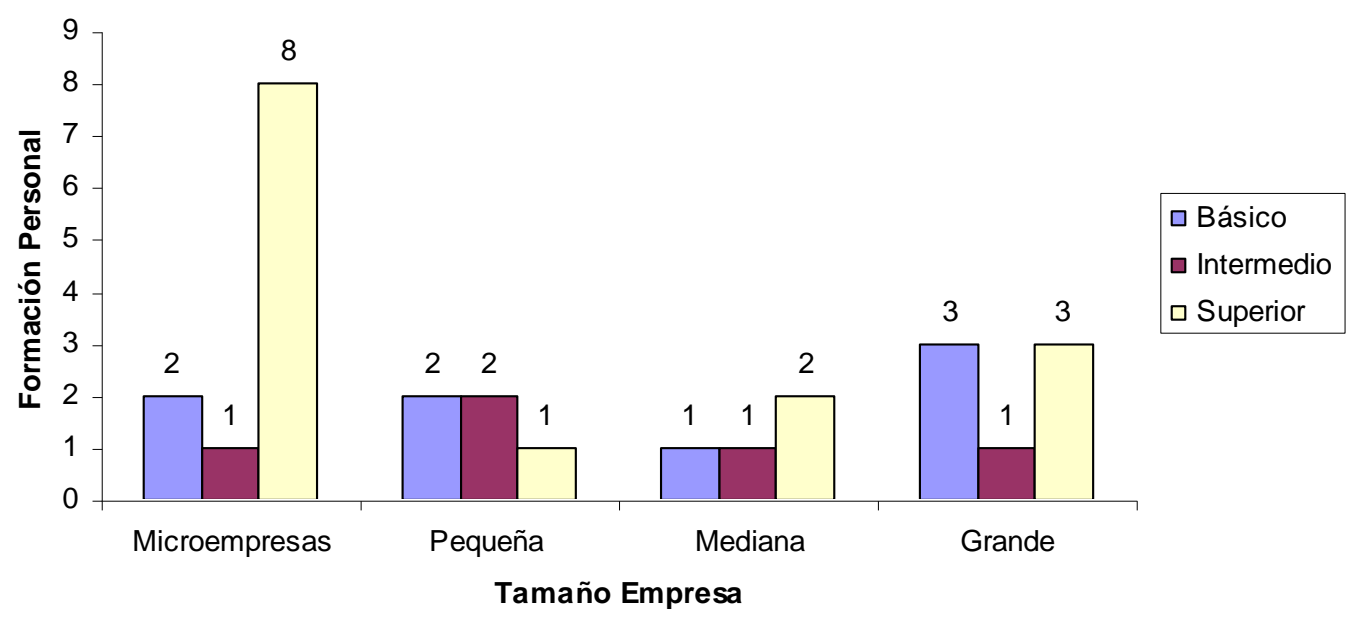

Fig. 1. Formación en SyS del personal en función del tamaño de la empresa. Fuente: Elaboración propia. 


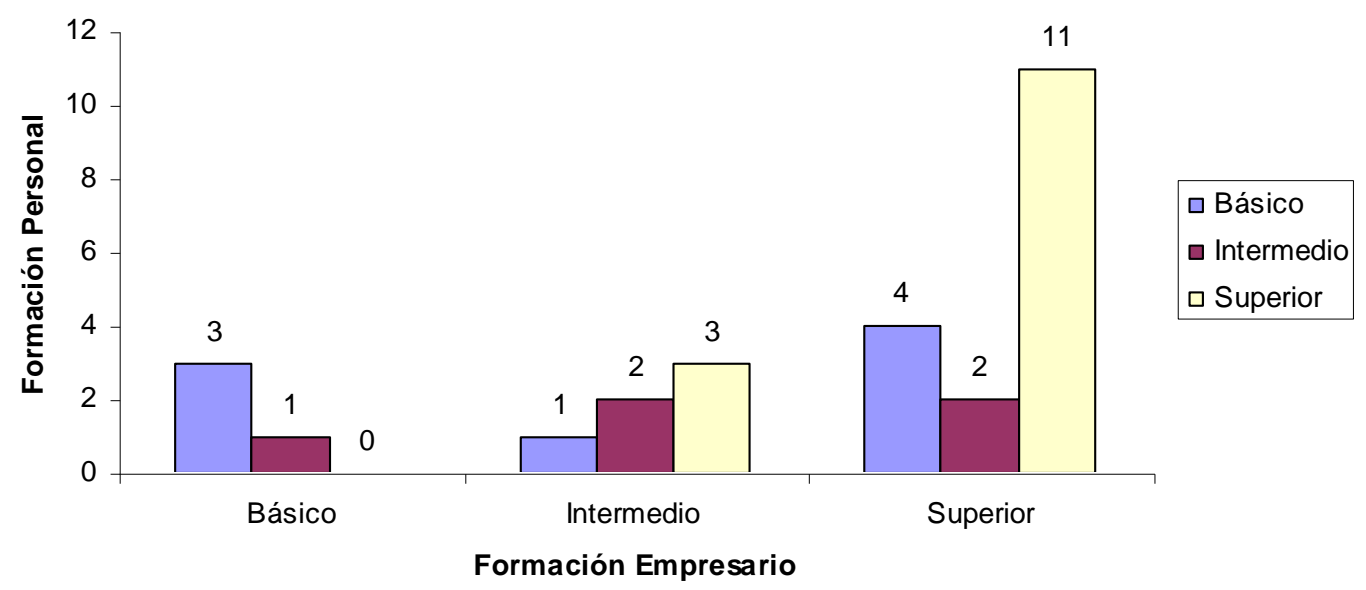

Fig. 2. Formación en SyS del personal en función de la formación en SyS del empresario. Fuente: Elaboración propia.

entre la formación del empresario y la del personal de la empresa, a mayor formación del empresario hay una mayor formación del personal de la empresa.

En el tercer bloque se analizaron los aspectos relativos a la gestión de la documentación de Seguridad y Salud necesaria durante la ejecución de la obra. En primer término se analizó el ítem correspondiente a la elaboración del PSS. Dos empresas pertenecientes al grupo Microempresas respondieron "No saben o no lo han realizado nunca", quince empresas los realizan con sus propios medios y diez empresas los subcontratan (cinco de ellas pertenecientes al sector Microempresas).

Respecto al ítem correspondiente al Libro de Subcontratación, ocho empresas respondieron "No sabe diligenciarlo o no lo ha diligenciado nunca", siete pertenecientes al grupo Microempresas y una al grupo Pequeña empresa. Once empresas lo cumplimentan con sus propios medios y ocho empresas subcontratan su elaboración (3 Micro, 2 Pequeñas, 2 Medianas y 1 Grande).

En el ítem referido a los trámites a realizar con la Administración responsable en materia de Seguridad y Salud, dos de las empresas indicaron "No saben o no los han realizado nunca", dieciocho empresas los realizan con sus propios medios y siete los subcontratan (2 Micro, 2 Pequeña, 2 Mediana y 1 Grande).

Si se analizan los resultados respecto a estos tres ítems se puede comprobar como: la mayoría de las empresas realizan los trámites con la administración por sus propios medios, la dificultad por parte de las microempresas a la hora de redactar documentos técnicos más complejos como el PSS y cómo, a pesar de qué es requerimiento normativo desde el 2007, la elaboración del Libro de Subcontratación es todavía un aspecto en el que las empresas o no saben realizarlo o tienen que recurrir a la ayuda de asesorías o técnicos externos, siendo especialmente significativo en el grupo Microempresas.
Finamente se realizaron dos cuestiones de respuesta múltiple sobre los Recursos Preventivos de las empresas contratistas y de las empresas subcontratistas. El nombramiento del personal como RP, a pesar de ser un requisito normativo desde 2004, tanto en empresas contratistas como subcontratistas, no se realiza por iniciativa propia sino que se realiza en la mayoría de las ocasiones por requerimiento del CSS. Otro aspecto remarcable que afecta al ámbito de las Microempresas, es que la figura del RP suele ser el gerente de la empresa, y que en el $42 \%$ de las empresas se indica que no está presente en la obra durante toda la jornada laboral, aspecto que contradice la propia definición de la figura.

\section{CONCLUSIÓN.}

La gestión de la Seguridad y Salud en las empresas de construcción es un factor clave en la prevención de accidentes. En el sector construcción español, y por ende valenciano, las microempresas y las pequeñas empresas conforman el mayor número de empresas, y también presentan los mayores datos de siniestrabilidad.

Entre las posibles causas están las deficiencias organizativas y el desconocimiento en materia de prevención de riesgos laborales, tal y como muestran los resultados de este estudio, donde todavía hay empresas que no integran la prevención de riesgos laborales en las políticas empresariales y un gran porcentaje de empresas organizan la actividad preventiva mediante la asunción por parte del empresario.

El nivel de formación de Seguridad y Salud está asociado tanto al tamaño de la empresa como al nivel de formación del responsable de ésta, cuando mayor es el tamaño de la empresa o cuando mayor es la formación del responsable de ésta, mayor es la formación del personal de la empresa.

La normativa sobre Seguridad y Salud en el sector construcción español ha intentado progresivamente corregir las malas praxis del sector, pero tal y como muestran los datos 
del estudio, ítems como los recursos preventivos, el REA o el LS siguen siendo objeto de confusión o de dificultad, en especial para las microempresas.

Dada la importancia de una adecuada Gestión de Seguridad y Salud para reducir el número de accidentes, por el perfil mayoritario de las empresas constructoras españolas (y valencianas), desde las administraciones se deberían potenciar políticas de concienciación y formación práctica obligatoria a todos los niveles jerárquicos en prevención de riesgos laborales.

\section{REFERENCIAS}

Ardite, D. \& Chotibhongs, R. (2005). Issues in subcontracting practice. Journal of Construction Engineering and Management, ASCE 131(8), 866-876.

Camino-López, M.A., Ritzel, D.O., Fontaneda, I. \& GonzálezAlcántara, O.J. (2008). Construction industry accidents in Spain. Journal of Safety Research, 39, 497-507.

Debrah Y.A. \& Ofori, G. (2001). Subcontracting foreign workers and job safety in the Singapore construction industry. Asia Pacific Business Review, 1-8, 145-166.

España. Ley 31/1995, de 8 de Noviembre, de Prevención de Riesgos Laborales. Boletín Oficial del Estado, 10 de Noviembre de 1995, núm. 269, 32590-32611.

España. Real Decreto 39/1997, de 17 de Enero, por el que se aprueba el Reglamento de los Servicios de Prevención. Boletín Oficial del Estado, 31 de Enero de 1997, núm. 27, 3031-3045.

España. Real Decreto 1627/1997, de 24 de Octubre, por el que se establecen disposiciones mínimas de seguridad y salud en las obras de construcción. Boletín Oficial del Estado, núm. 256, 30875-30888.

España. Real Decreto 171/2004, de 30 de Enero, por el que se desarrolla el artículo 24 de la Ley 31/1995, de 8 de Noviembre, de Prevención de Riesgos Laborales, en materia de coordinación de actividades empresariales. Boletín Oficial del Estado, 31 de Enero de 2004, núm. 27, 4160-4165.

España. Ley 32/2006, de 18 de Octubre, reguladora de la subcontratación en el Sector de la Construcción. Boletín Oficial del Estado, 19 de Octubre de 2006, núm. 250, 36317-36323.

España. Real Decreto 1109/2007, de 24 de Agosto, por el que se desarrolla la Ley 32/2006, de 18 de Octubre, reguladora de la subcontratación en el Sector de la Construcción. Boletín Oficial del Estado, 25 de Agosto de 2007, núm. 204, 35747-35764.
Esteban-Gabriel, J. \& Martín-Pérez, I. (2015). Management analysis of Health and Safety on construction sites in South Africa. New construction regulations released, new challenges, new opportunities. Anales de Edificación, $1(1), 49-54$.

Fuentes-Bargues, J.L., Fuentes-Bargues, D. \& Verdejo-López, Y. (2014). Estudio sobre la organización y gestión de seguridad y salud en obras de construcción. Libro de Actas del XVIII Congreso Internacional de Ingeniería de Proyectos, Alcañiz, Spain, 1943-1953.

Fuentes-Bargues, J.L., González-Gaya, C. \& González-Cruz, M.C. (2015). La contratación pública de obras: situación actual y puntos de mejora. Informes de la Construcción, 67(537): doi://dx.doi.org/10.3989/ ic.12.130.

Health and Safety Executive (HSE). Blackspot construction: a study of five years of fatal accidents in the building and civil engineering industries. London: HMSO; 1988.

Huang, X. \& Hinze, J. (2003). Analysis of construction worker fall accidents. Journal Construction Engineering Management, 129, 262-271.

Lin, J. \& Mills, A. (2001). Measuring the occupational health and safety performance of construction companies in Australia. Facilities, 19(3-4), 131-139.

Loosemore, N. \& Andonakis, N. (2007). Barriers to implementing OHS reforms - The experience of small subcontractors in the Australian Construction Industry. International Journal of Project Management, 25, 579588.

López-Alonso, M., Ibarrondo-Dávila, P. \& Rubio-Gámez, M.C. (2015). Análisis de los costes de prevención en obras de construcción. Informes de la construcción, 67(537): eo55, doi://dx.doi.org/10.3989/ic.13.062.

López-González, L.M. \& López-Ochoa, L.M. (2012). La seguridad en el sector de la construcción desde la perspectiva de los proyectistas riojanos. Libro de Actas del XVI Congreso Internacional de Ingeniería de Proyectos, Valencia, Spain, 627-637.

López-González, L.M., López-Ochoa, L.M., ZamaollaMadariaga, L. \& Sáenz-Díez, F. (2013). La seguridad en el sector de la construcción desde la perspectiva de directores de obra riojanos. Libro de Actas del XVII Congreso Internacional de Ingeniería de Proyectos, Logroño, Spain, 552-562.

Mattila, M., Rantanen, E. \& Hyttinen, M. (1994). The quality of work environment, supervision and safety in building construction. Safety Science, 17-4, 321-329. 
Ng S.T. \& Tang Z. (2010) Labour-intensive construction subcontractors: Their critical success factor. International Journal of Project Management, 28, 732-740.

Pellicer Armiñana, T.M. (2003). La Gestión de las Empresas Constructoras: Análisis, Diseño y Desarrollo de un Modelo de Control. PhD Thesis, Universitat Politècnica de València, Spain.

Pérez-Alonso, J., Carreño-Ortega, A., Vázquez-Cabrera, F.J. \& Callejón-Ferre, A.J. (2012). Accidents in the greenhouse-construction industry of SE Spain. Applied Ergonomics, 43, 69-80.

Reynolds, J.R., Tutesigensi, A. \& Lindsell, D.J. (2008). An evaluation of health and safety management in small construction enterprises in the United Kingdom. In: Hinze, J., Bohner, S. and Lew, J., (eds). Proceedings of CIB W99 14th Rinker International Conference. Evolution of and developments in construction safety and health. CIB W99 14th Rinker International Conference, 911 March, 2008, Gainesville, Florida, USA, 541-550.

Ros-Serrano, A., Ortiz-Marcos, I., Palomo-Sánchez, J.G. \& Uruburu-Colsa, A. (2013). A proposal for improving safety in construction projects by strengthening coordinators'competencies in health and safety issues. Safety Science, 54, 92-103.

Sesé, A., Palmer, A.L., Cajal, B., Montaño, J.J., Jiménez, R. \& Llorens, N. (2002). Occupational safety and health in Spain. Journal of Safety Research, 33, 511-525.

Tam, C.M., Zeng, S.X. \& Deng, Z.M. (2004). Identifying elements of poor construction safety management in China. Safety Science, 42(7), 569-586.

Teo, E.A.L., Ling, F.Y.Y. \& Chong, A.F.W. (2005). Framework for project managers to manage construction safety. International Journal of Project Management, 23, 329-341.

Ulubeyli, S., Kazaz, A. \& Er, B. (2015). Health and safety perception of workers in Turkey: a survey of construction sites. International Journal of Occupational Safety and Ergonomics, 20-2, 323-338.

Unión Europea. Directiva 89/391/CEE del Consejo, de 12 de Junio de 1989, relativa a la aplicación de medidas para promover la mejora de la seguridad y salud de los trabajadores en el trabajo. Diario Oficial de la Unión Europea, 29 de Junio de 1989, núm. L183, 1-8. 